\title{
Cost-Effectiveness Findings from the Agewell Pilot Study of Behaviour Change to Promote Health and Wellbeing in Later Life
} \author{
Clare $^{2}$ \\ ${ }^{1}$ Centre for Health Economics and Medicines Evaluation, IMSCaR, Bangor University, UK \\ ${ }^{2} R E A C H$ : The Centre for Research in Ageing and Cognitive Health, School of Psychology, University of Exeter, UK \\ ${ }^{3}$ Wales Institute of Social and Economic Research, Data and Methods, Cardiff University, UK \\ ${ }^{4}$ North Wales Organisation for Randomised Trials in Health (NWORTH), Bangor University, UK \\ ${ }^{5}$ Faculty of Medicine, University of New South Wales, Australia \\ ${ }^{6}$ School of Sports, Health and Exercise Sciences, Bangor University, UK
}

Carys L Jones ${ }^{1 *}$, Rhiannon Tudor Edwards ${ }^{1}$, Sharon M Nelis ${ }^{2}$, lan R Jones ${ }^{3}$, John V Hindle ${ }^{4}$, Jeanette M Thom ${ }^{5}$, Jennifer Cooney ${ }^{6}$ and Linda

\begin{abstract}
Background: Participation in cognitive and physical activities may help to maintain health and wellbeing in older people. The Agewell study explored the feasibility of increasing cognitive and physical activity in older people through a goal-setting approach. This paper describes the findings of the cost-effectiveness analysis.

Method: Individuals over the age of 50 and attending an Agewell centre in North Wales were randomised to one of three conditions: control (IC), goal-setting (GS), or goal-setting with mentoring (GM). We undertook a cost-effectiveness analysis comparing GS vs. IC, GM vs. IC and GM vs. GS. The primary outcome measure for this analysis was the QALY, calculated using the EQ-5D. Participants' health and social care contacts were recorded and costed using national unit costs.

Results: Seventy participants were followed-up at 12 months. Intervention set up and delivery costs were $£ 252$ per participant in the GS arm and £269 per participant in the GM arm. Mean health and social care costs over 12 months were $£ 1,240$ (s.d. £3,496) per participant in the IC arm, £1,259 (s.d. £3,826) per participant in the GS arm and £1,164 (s.d. £2,312) per participant in the GM arm. At a willingness to pay threshold of $£ 20,000$ per QALY there was a $65 \%$ probability that GS was cost-effective compared to IC (ICER of $£ 1,070)$. However, there was only a $41 \%$ probability that GM was cost-effective compared to IC (ICER of $£ 2,830$ ) at a threshold of $£ 20,000$ per QALY.

Conclusion: Setting up and running the community based intervention was feasible. Due to the small sample size it is not possible to draw a firm conclusion about cost-effectiveness; however, our preliminary results suggest that goalsetting is likely to be cost-effective compared to the control condition of no goal-setting, the addition of mentoring was effective but not cost-effective.
\end{abstract}

\section{Keywords: Cost; Ageing; Goal-setting; Active}

\section{Introduction}

Life expectancy in the United Kingdom has increased over time and more people are living longer [1]. Older people are at risk of social isolation, which is associated with higher rates of depression and unmet health care needs [2,3]. Zaninotto et al. determined that quality of life was higher in older people who had an absence of depression, absence of limiting illnesses, were in the richest quintile of wealth, were in employment, were cohabiting and were in the $80^{\text {th }}$ percentile for a positive social network [4]. A review of health promotion strategies to prevent social isolation in older people found key determinants to consider when addressing social isolation were income and social status, personal health practices and coping skills and social support networks [5]. As well as affecting quality of life negatively, social isolation in older people is also believed to influence cognitive decline [6]. Alongside social engagement, participating in cognitive and physical activities may contribute to dementia prevention and risk reduction, thus there is a need to identify ways to promote and increase cognitive and physical activity in older people within a context of social participation [7]. A recent review of interventions aiming to increase physical activity in older people concluded that individually tailored interventions with defined personal goals may be more effective, however the review did not establish the evidence base for the costeffectiveness of physical activity interventions [8]. This paper describes the cost of setting up and delivering the Agewell community-based goal-setting intervention to increase activity in older people, along with cost-effectiveness findings. Effectiveness findings are published in Clare et al. [9].

\section{Method}

\section{Study design}

Full details of the Agewell study protocol are published as Clare et al. [10]. Agewell was a pilot randomised trial aiming to assess the effect of three different types of interview on subsequent behaviour change. The three arms of the pilot trial were a control condition (IC), a goalsetting condition (GS) and a goal-setting with mentoring condition (GM). Assessments were carried out by blinded researchers at baseline and twelve months. Randomisation was conducted by the North Wales Organisation for Randomised Trials in Health (NWORTH) using a

${ }^{*}$ Corresponding author: Carys Jones, Centre for Health Economics and Medicines Evaluation, IMSCaR, Bangor University, UK, Tel: +44 (0)1248 382483; Fax: +44 (0)1248 383982; E-mail: c.l.jones@bangor.ac.uk

Received November 13, 2015; Accepted December 01, 2015; Published December 08, 2015

Citation: Jones CL, Edwards RT, Nelis SM, Jones IR, Hindle JV, et al (2015) Cost-Effectiveness Findings from the Agewell Pilot Study of Behaviour Change to Promote Health and Wellbeing in Later Life. Health Econ Outcome Res Open Access 1: 105. doi: 10.4172/2471-268x/1000105

Copyright: $\odot 2015$ Jones CL, et al. This is an open-access article distributed under the terms of the Creative Commons Attribution License, which permits unrestricted use, distribution, and reproduction in any medium, provided the original author and source are credited. 
dynamic adaptive computer algorithm. Randomisation was stratified by gender and married couples were randomised together to the same study arm to avoid cross-contamination. The AgeWell study was approved by the School of Psychology Research Ethics Committee at Bangor University.

\section{Study population}

Individuals aged 50 years and over living in Nefyn, Gwynedd (North Wales) and attending the Nefyn Agewell Centre were invited to participate. No exclusion criteria were placed on individuals. Individuals attending the centre who did not wish to participate in the study were able to attend the centre as usual.

\section{Setting}

The intervention was conducted in partnership with Age Cymru Gwynedd a Môn (ACGM). ACGM runs the Agewell Centre in Nefyn, Gwynedd, which was set up as part of the research project. The Agewell Centre is based in the village community centre and led by an AGCM co-ordinator, supported by a volunteer management group of centre attendees. The centre is open three days a week to provide social contact and a range of meaningful and enjoyable activities for over 50s. The program included a range of classes: exercise, computer, history and cookery. A range of other activities were also available inducing arts and crafts, bowling, Nordic walking, theatre trips, and photography.

\section{Intervention}

Participants randomised to the IC arm received an interview consisting of a general discussion about the centre facilities. Participants randomised to the GS arm received a structured goal-setting interview. Participants randomised to the GM arm received a structured goalsetting interview supplemented by five mentoring telephone calls over the course of the one-year follow-up period.

\section{Outcome measures for cost-effectiveness analysis}

A comprehensive list of outcome measures used in the Agewell study is available in the published protocol [10]. The primary outcome measure for the cost-effectiveness analysis was the quality-adjusted life year (QALY), calculated using the EQ-5D. The EQ-5D is a generic, preference based instrument for measuring health-related quality of life [11]. A scoring algorithm was used to convert responses into an index value of between -0.59 and 1 , with 1 representing best possible health- related quality of life [12].

\section{Measurement of intervention costs}

The intervention was conducted at the Agewell centre, with centre set-up costs annuitized over 3 years. Staff time developing the intervention was classed as a set-up cost, and annuitized over 3 years. Staff time delivering the initial interviews in all three conditions, and conducting on-going mentoring phone calls in the goal-setting with mentoring condition, was costed. It was not necessary to discount intervention costs as the follow-up period was one year.

\section{Measurement of health and social care costs}

An adapted Client Service Receipt Inventory (CSRI) [13] was used to record participants' contacts with primary and secondary health and social care services. National unit costs for the price year 2011-2012 were assigned to services accessed, to calculate a mean total cost per participant $[14,15]$. Service use costs were not discounted as the followup period was one year.

\section{Economic evaluation}

We undertook a cost-effectiveness analysis comparing GS vs IC, GM vs IC and GM vs GS. A public sector, multi-agency perspective was adopted for the economic evaluation. Cost-effectiveness analysis was conducted in line with MRC guidelines for the evaluation of complex interventions [16], and with our standard operating procedure for economic evaluations alongside randomised controlled trials [17]. The base case analysis was an intention-to-treat cost-utility analysis using the QALY. Non-parametric bootstrapping with 1000 replications [18] was used to address the uncertainty associated with point estimates of cost effectiveness ratios. To explore whether the results were robust to our assumptions, a sensitivity analysis was conducted to test the potential effect of the goal-setting interviews and mentoring being delivered by an ACGM staff member on an annual salary of $£ 12,150$.

\section{Results}

Seventy-five participants were randomised into the three conditions. At the 12 month follow-up, 70 participants completed measures for both the clinical-effectiveness and cost-effectiveness analysis (IC $n=27, G S n=21, G M n=22$ ), a retention rate of $93 \%$.

\section{Baseline data}

Normality tests on baseline data indicated that the EQ-5D and cost data were not normally distributed, so the non-parametric Kruskal-Wallis test was used to test for significant differences between conditions. No significant differences existed at baseline ( $\mathrm{p}=0.193$ for costs, $\mathrm{p}=0.905$ for EQ-5D;), so follow-up data used in the economic analysis were not adjusted for baseline values (Table 1).

Mean EQ-5D index scores at baseline were between 0.862 and 0.899 . The UK population norm for this age group is 0.78 (s.d. 0.26 ) [19], indicating that Agewell participants had comparable health to the general population.

\section{Health and social care use over the study period}

Table 2 shows health and social care contacts occurring between baseline and 12 month follow-up for the 70 participants who were assessed at both time-points. The mean cost per participant for health and social care contacts was $£ 1,240.23$ (s.d. $£ 3,495.88$ ) in the control condition, $£ 1,258.66$ (s.d. $£ 3,825.71$ ) in the goal-setting condition and $£ 1,163.89$ (s.d. $£ 2,312.49$ ) in the goal-setting with mentoring condition. The difference in costs between conditions was not significant $(\mathrm{p}=0.806)$ (Table 2).

\section{Other activities}

Data on activities undertaken by participants outside of the Agewell centre during the study period were collected. This service use was low; 2 participants were enrolled on the local authority funded patient referral physical activity scheme and 1 participant was undertaking an unspecified local authority funded activity. These activities were not included in the cost analysis.

\section{Intervention costs}

Table 3 summarises the cost of setting up and running the Agewell centre and setting up and delivering the intervention. The costs of setting up the Agewell centre were annuitized over 3 years and included an ACGM development officer employed at 50\% FTE, equipment costs, volunteer training, administration, promotion and recruitment costs. Based on attendance figures from the study period we estimated that up to 600 people would attend the centre during a 3 year time frame; 
Citation: Jones CL, Edwards RT, Nelis SM, Jones IR, Hindle JV, et al (2015) Cost-Effectiveness Findings from the Agewell Pilot Study of Behaviour Change to Promote Health and Wellbeing in Later Life. Health Econ Outcome Res Open Access 1: 105. doi: 10.4172/2471-268x/1000105

Page 3 of 6

\begin{tabular}{|c|c|c|c|}
\hline & $\begin{array}{c}\text { IC }(n=27) \\
\text { Mean (s.d.) }\end{array}$ & $\begin{array}{l}\text { GS }(n=24) \\
\text { Mean (s.d.) }\end{array}$ & $\begin{array}{l}\text { GM }(n=24) \\
\text { Mean (s.d.) }\end{array}$ \\
\hline Gender: Female (n, \%) & $23(85.2 \%)$ & $23(95.8 \%)$ & $19(79.2 \%)$ \\
\hline Age & $70.22(7.77)$ & $67.50(7.66)$ & $66.67(8.19)$ \\
\hline Years of education & $12.70(2.91)$ & $13.79(3.18)$ & $13.58(2.68)$ \\
\hline Living situation: living with others & $15(55.6 \%)$ & $16(66.7 \%)$ & $18(75.0 \%)$ \\
\hline Social capital: good & $8(29.6 \%)$ & $9(37.5 \%)$ & $8(33.3 \%)$ \\
\hline Social capital: poor & $19(70.4 \%)$ & $15(62.5 \%)$ & $16(66.7 \%)$ \\
\hline Material deprivation: none & $10(37.0 \%)$ & $7(29.2 \%)$ & $2(8.3 \%)$ \\
\hline Material deprivation: moderate & $12(44.4 \%)$ & $13(54.2 \%)$ & $15(62.5 \%)$ \\
\hline Material deprivation: high & $5(18.5 \%)$ & $4(16.7 \%)$ & $7(29.2 \%)$ \\
\hline EQ-5D index value & $0.899(0.141)$ & $0.891(0.133)$ & $0.862(0.227)$ \\
\hline Subjective health: poor/ very poor & 0 & 0 & $2(8.3 \%)$ \\
\hline Subjective health: not too good & $4(14.8 \%)$ & $3(12.5 \%)$ & 0 \\
\hline Subjective health: good/ very good & $23(85.2 \%)$ & $21(87.5 \%)$ & $22(91.7 \%)$ \\
\hline $\begin{array}{c}\text { Health and social care contact cost for the } 3 \text { months before } \\
\text { baseline }\end{array}$ & $£ 255.84(£ 374.78)$ & $£ 169.96(£ 250.47)$ & $£ 370.96(£ 1,406.69)$ \\
\hline
\end{tabular}

Note: IC: Control, GS: Goal-Setting, GM: Goal-Setting With Mentoring.

Table 1: Baseline characteristics for the full sample.

\begin{tabular}{|c|c|c|c|c|c|c|c|c|c|c|c|c|}
\hline & \multicolumn{4}{|c|}{ Control $(n=27)$} & \multicolumn{4}{|c|}{ Goal-setting $(n=21)$} & \multicolumn{4}{|c|}{ Goal-setting with mentoring $(n=22)$} \\
\hline & $\mathrm{N}(\%)$ & $\begin{array}{c}\text { Total } \\
\text { contacts }\end{array}$ & Total cost & $\begin{array}{l}\text { Mean cost per } \\
\text { participant (SD) }\end{array}$ & $\mathrm{N}(\%)$ & $\begin{array}{c}\text { Total } \\
\text { contacts }\end{array}$ & Total cost & $\begin{array}{l}\text { Mean cost per } \\
\text { participant (SD) }\end{array}$ & $\mathrm{N}(\%)$ & $\begin{array}{l}\text { Total } \\
\text { contacts }\end{array}$ & Total cost & $\begin{array}{l}\text { Mean cost per } \\
\text { participant (SD) }\end{array}$ \\
\hline $\begin{array}{c}\text { General } \\
\text { practitioner }\end{array}$ & $24(88.9 \%)$ & 108 & $£ 7,388$ & $£ 273.63(£ 355.30)$ & $17(81.0 \%)$ & 63 & $£ 4,073$ & $£ 193.95(£ 132.61)$ & $19(86.4 \%)$ & 48 & $£ 3,024$ & $£ 137.45(£ 107.61)$ \\
\hline Practice nurse & $25(92.6 \%)$ & 58 & $£ 812$ & $£ 30.07(£ 28.72)$ & $19(90.5 \%)$ & 77 & $£ 1,078$ & $£ 51.33(£ 75.57)$ & $15(68.2 \%)$ & 43 & $£ 602$ & $£ 27.36(£ 40.64)$ \\
\hline District nurse & $2(7.4 \%)$ & 4 & $£ 244$ & $£ 9.04(£ 35.61)$ & $1(4.8 \%)$ & 4 & $£ 280$ & $£ 13.33(£ 61.10)$ & $3(13.6 \%)$ & 9 & $£ 618$ & $£ 28.09(£ 104.88)$ \\
\hline Counsellor & 0 & 0 & 0 & 0 & $1(4.8 \%)$ & 1 & $£ 60$ & $£ 2.86(£ 13.09)$ & 0 & 0 & 0 & 0 \\
\hline Physiotherapist & $6(22.2 \%)$ & 47 & $£ 1,551$ & $£ 57.44(£ 150.28)$ & $4(19.0 \%)$ & 24 & $£ 792$ & $£ 37.71$ (£84.32) & $5(22.7 \%)$ & 60 & $£ 1,980$ & $£ 90.00(£ 243.18)$ \\
\hline $\begin{array}{c}\text { Occupational } \\
\text { health therapist }\end{array}$ & 0 & 0 & 0 & 0 & 0 & 0 & 0 & 0 & $1(4.5 \%)$ & 1 & $£ 33$ & $£ 1.50(£ 7.04)$ \\
\hline Chiropodist & $7(25.9 \%)$ & 21 & $£ 630$ & $£ 23.33(£ 43.50)$ & $4(19.0 \%)$ & 19 & $£ 570$ & $£ 27.14$ (£81.56) & $2(9.1 \%)$ & 12 & $£ 360$ & $£ 16.36(£ 59.80)$ \\
\hline Dietician & $2(7.4 \%)$ & 8 & $£ 272$ & $£ 10.07(£ 46.02)$ & $1(4.8 \%)$ & 8 & $£ 272$ & $£ 12.95$ (£59.36) & 0 & 0 & 0 & 0 \\
\hline Dentist & $1(3.7 \%)$ & 2 & $£ 50$ & $£ 1.85(£ 9.62)$ & $3(14.3 \%)$ & 5 & $£ 125$ & $£ 5.95(£ 15.62)$ & $1(4.5 \%)$ & 1 & $£ 25$ & $£ 1.14(£ 5.33)$ \\
\hline Optician & $1(3.7 \%)$ & 1 & $£ 21$ & $£ 0.78(£ 4.04)$ & 0 & 0 & 0 & 0 & $3(13.6 \%)$ & 3 & $£ 63$ & $£ 2.86(£ 7.38)$ \\
\hline $\begin{array}{l}\text { Alternative } \\
\text { therapist }\end{array}$ & $2(7.4 \%)$ & 3 & $£ 51$ & $£ 1.89(£ 7.20)$ & 0 & 0 & 0 & 0 & $1(4.5 \%)$ & 8 & $£ 136$ & $£ 6.18(£ 29.00)$ \\
\hline $\begin{array}{c}\text { Gynaecology } \\
\text { nurse }\end{array}$ & 0 & 0 & 0 & 0 & $1(4.8 \%)$ & 3 & $£ 243$ & $£ 11.57(£ 53.03)$ & 0 & 0 & 0 & 0 \\
\hline $\begin{array}{l}\text { Total community } \\
\text { service use }\end{array}$ & - & 252 & $£ 11,019$ & $£ 408.11(£ 417.60)$ & - & 204 & $£ 7,493$ & $£ 356.81(£ 357.75)$ & - & 185 & $£ 6,841$ & $£ 310.95(£ 372.37)$ \\
\hline Outpatient service & $14(51.9 \%)$ & 48 & $£ 5,088$ & $£ 188.44(£ 279.42)$ & $10(47.6 \%)$ & 20 & $£ 2,120$ & $£ 100.95(£ 131.87)$ & $12(54.5 \%)$ & 17 & $£ 1,802$ & $£ 81.91$ (£103.09) \\
\hline Inpatient nights & $3(11.1 \%)$ & 28 & $£ 17,379$ & $\begin{array}{c}£ 643.67 \\
(£ 3,032.70)\end{array}$ & $1(4.8 \%)$ & 21 & $£ 16,728$ & $\begin{array}{c}£ 796.56 \\
(£ 3,650.30)\end{array}$ & $4(18.2 \%)$ & 14 & $£ 16,872$ & $\begin{array}{c}£ 766.89 \\
(£ 2,024.62)\end{array}$ \\
\hline $\begin{array}{l}\text { Accident and } \\
\text { Emergency }\end{array}$ & 0 & 0 & 0 & 0 & $1(4.8 \%)$ & 1 & $£ 91$ & $£ 4.33(£ 19.86)$ & $1(4.5 \%)$ & 1 & $£ 91$ & $£ 4.14(£ 19.40)$ \\
\hline Total hospital use & - & - & $£ 22,467$ & $\begin{array}{c}£ 832.12 \\
(£ 3,159.32)\end{array}$ & - & - & $£ 18,939$ & $\begin{array}{c}£ 901.85 \\
(£ 3,725.00)\end{array}$ & - & - & $£ 18,765$ & $\begin{array}{c}£ 852.94 \\
(£ 2,093.18)\end{array}$ \\
\hline TOTAL & - & - & $£ 33,486$ & $\begin{array}{c}£ 1,240.23 \\
(£ 3,495.88)\end{array}$ & - & - & $£ 26,432$ & $\begin{array}{c}£ 1,258.66 \\
(£ 3,825.71)\end{array}$ & - & - & $£ 25,606$ & $\begin{array}{c}£ 1,163.89 \\
(£ 2,312.49)\end{array}$ \\
\hline
\end{tabular}

Table 2: Health and social care contacts in the 12 months following baseline. 
Citation: Jones CL, Edwards RT, Nelis SM, Jones IR, Hindle JV, et al (2015) Cost-Effectiveness Findings from the Agewell Pilot Study of Behaviour Change to Promote Health and Wellbeing in Later Life. Health Econ Outcome Res Open Access 1: 105. doi: 10.4172/2471-268x/1000105

Page 4 of 6

thus we divided the total cost by 600 to estimate the cost per person. Centre running costs included staff costs, rent and administration costs e.g. utility bills.

Developing the intervention took 65 hours. Training staff to deliver the interviews (both control and goal-setting) took 10 hours. In the intervention delivery, the baseline interview was conducted by a trained researcher. The average length of interviews for participants in the control condition was 60 minutes. The average length of interviews for participants in the intervention conditions (GS, GM) was 90 minutes. Participants in the GM condition received an average of 5 mentoring phone calls, lasting approximately 10 minutes per call (Table 3).

\section{Cost to participants}

Centre attendees were asked for a financial contribution to cover the cost of hiring external facilitators, regardless of whether they were trial participants or not. Each attendee paid $£ 2.50$ per session for activities such as tai chi, line dancing, ballroom dancing, calligraphy, choir, pottery, exercise class and Nordic walking. The cost of healthy eating and arts classes was slightly lower at $£ 2.00$ per person. During the study period, trial participants paid a mean of $£ 59.55$ per person for session fees (s.d. $£ 77.32$, range $£ 0-£ 272.50$ ).

\section{Cost-effectiveness analysis}

The mean costs (including intervention costs) and QALYs accrued between baseline and 12 months for each trial arm are shown in Table 4. In this pilot study a small incremental QALY gain was seen between GS and IC, and the incremental cost-effectiveness ratio (ICER) between conditions was $£ 1,070$ (Table 4).

Figure 1 shows the cost-effectiveness planes for the GS and GM conditions compared to the control condition. The majority of plots in the GS cost-effectiveness plane fall in the East of the figure, suggesting that GS is more effective than the control condition. More plots fall in the West of the cost-effectiveness plane for GM compared to control (Figure 1).

Cost-effectiveness acceptability curves indicate the probability that an intervention is cost-effective at a range of willingness to pay thresholds. The cost-effectiveness acceptability curve in Figure 2 indicates a $65 \%$ probability that GS was cost-effective compared to the control condition at the NICE threshold of $£ 20,000$ per QALY [20]. However, the cost-effectiveness acceptability curve for GM compared to control indicated only a $41 \%$ probability of being cost-effective compared to the control condition at a threshold of $£ 20,000$ per QALY. Comparing GM to GS, there was a $36 \%$ probability that GM was costeffective at a threshold of $£ 20,000$ per QALY (Figure 2).

\section{Sensitivity analysis}

In the base case analysis, the goal-setting interviews and mentoring calls were conducted by a researcher on an annual salary of approximately $£ 33,000$. We conducted a sensitivity analysis to explore the effect of the goal-setting interviews and mentoring being delivered by an ACGM staff member on an annual salary of approximately $£ 12,000$. In this scenario the cost per participant (including centre costs and intervention costs) was $£ 228.94$ in the control condition, $£ 233.18$ in the goal-setting condition and $£ 240.25$ in the goal-setting with mentoring condition (Table 5).

\section{Discussion}

The Agewell pilot study explored the feasibility of setting up and

\begin{tabular}{|l|l|l|}
\hline & $\begin{array}{l}\text { Total } \\
\text { annuitized } \\
3 \text { years }\end{array}$ & $\begin{array}{l}\text { cost, } \\
\text { over person }\end{array}$ \\
\hline One-off centre set-up cost & $£ 11,600.14$ & $£ 19.33^{1}$ \\
\hline Annual cost of running the centre & $£ 38,122.44$ & $£ 190.61^{2}$ \\
\hline Intervention development and set-up cost & $£ 862.62$ & $£ 11.50^{3}$ \\
\hline 60 minute control interview cost per person- IC arm - & $£ 20.32$ \\
\hline 90 minute goal-setting interview cost per person- - & $£ 30.48$ \\
\hline GS arm & $£ 47.41$ \\
\hline 90 minute goal-setting interview and mentoring - & \\
\hline Cost per person- GM arm & & \\
\hline Total cost per participant, including centre and intervention costs & \\
\hline IC arm & $£ 241.77$ \\
\hline GS arm & $£ 261.93$ \\
\hline GM arm & & \\
\hline
\end{tabular}

IC: Control, GS: Goal-Setting, GM: Goal-Setting With Mentoring

${ }^{1}$ Based on up to 600 people attending over 3 years

${ }^{2}$ Based on up to 200 people attending per year

${ }^{3}$ Based on the 75 participants randomised in the study

Table 3: Costs of setting up and running the centre and intervention.

\begin{tabular}{|c|c|c|c|}
\hline & IC $(n=27)$ & GS $(n=21)$ & $\mathrm{GM}(\mathrm{n}=22)$ \\
\hline Mean (s.d.) cost & $\begin{array}{c}£ 1,482.00 \\
(£ 3,495.88)\end{array}$ & $\begin{array}{c}£ 1,510.59 \\
(£ 3,825.71)\end{array}$ & $\begin{array}{c}£ 1,432.75 \\
(£ 2,312.49)\end{array}$ \\
\hline \multirow[t]{2}{*}{ Mean (s.d.) QALY } & $0.8464(0.1849)$ & $0.8731(0.1670)$ & $0.8290(0.2442)$ \\
\hline & $\begin{array}{l}\text { GS v IC } \\
(95 \% \mathrm{CI})\end{array}$ & $\begin{array}{l}\mathrm{GM} \text { v IC } \\
(95 \% \mathrm{Cl})\end{array}$ & $\begin{array}{l}\mathrm{GM} \vee \mathrm{GS} \\
(95 \% \mathrm{CI})\end{array}$ \\
\hline Incremental cost & $\begin{array}{c}£ 28.58 \\
(-£ 2,045.86 \text { to } \\
£ 1,957.86)\end{array}$ & $\begin{array}{c}-£ 49.25 \\
(-£ 1,728.65 \text { to } \\
£ 1,528.18)\end{array}$ & $\begin{array}{c}-£ 77.83 \\
(-£ 2,150.62 \text { to } \\
£ 1,540.99)\end{array}$ \\
\hline Incremental QALY & $\begin{array}{c}0.0267 \\
(-0.078 \text { to } 0.127)\end{array}$ & $\begin{array}{c}-0.0174 \\
(-0.156 \text { to } 0.092)\end{array}$ & $\begin{array}{c}-0.0441 \\
(-0.163 \text { to } 0.077)\end{array}$ \\
\hline ICER & $£ 1,070$ & $£ 2,830$ & $£ 1,765$ \\
\hline $\begin{array}{l}\text { Probability of cost- } \\
\text { effectiveness at } \\
£ 20,000 \text { per QALY }\end{array}$ & $65 \%$ & $41 \%$ & $36 \%$ \\
\hline $\begin{array}{l}\text { Probability of cost- } \\
\text { effectiveness at } \\
£ 30,000 \text { per QALY }\end{array}$ & $68 \%$ & $44 \%$ & $33 \%$ \\
\hline
\end{tabular}

Note: GS: Goal-Setting, GM: Goal-Setting with Mentoring, IC: Control

Table 4: Incremental costs and QALYs between conditions at 12 months.

\begin{tabular}{|c|c|c|c|}
\hline & $\begin{array}{c}\text { GS v IC } \\
(95 \% \mathrm{Cl})\end{array}$ & $\begin{array}{c}\mathrm{GM} \vee \mathrm{IC} \\
(95 \% \mathrm{CI})\end{array}$ & $\begin{array}{l}\mathrm{GM} \vee \mathrm{GS} \\
(95 \% \mathrm{Cl})\end{array}$ \\
\hline Incremental cost & $\begin{array}{c}£ 22.66 \\
(-£ 2,072.96 \text { to } \\
£ 2,365.28)\end{array}$ & $\begin{array}{c}-£ 65.03 \\
(-£ 1,792.80 \text { to } \\
£ 1,404.68)\end{array}$ & $\begin{array}{c}-£ 87.69 \\
(-£ 2,275.11 \text { to } \\
£ 1,492.09)\end{array}$ \\
\hline Incremental QALY & $\begin{array}{c}0.0267 \\
(-0.078 \text { to } 0.127)\end{array}$ & $\begin{array}{c}-0.0174 \\
(-0.156 \text { to } 0.092)\end{array}$ & $\begin{array}{c}-0.0441 \\
(-0.163 \text { to } 0.077)\end{array}$ \\
\hline ICER & $£ 849$ & $£ 3,737$ & $£ 1,988$ \\
\hline $\begin{array}{l}\text { Probability of cost- } \\
\text { effectiveness at } \\
£ 20,000 \text { per QALY }\end{array}$ & $65 \%$ & $44 \%$ & $32 \%$ \\
\hline $\begin{array}{l}\text { Probability of cost- } \\
\text { effectiveness at } \\
£ 20,000 \text { per QALY }\end{array}$ & $67 \%$ & $43 \%$ & $28 \%$ \\
\hline
\end{tabular}

Note: GS: Goal-Setting, GM: Goal-Setting with Mentoring, IC: Control Table 5: Sensitivity analysis. 

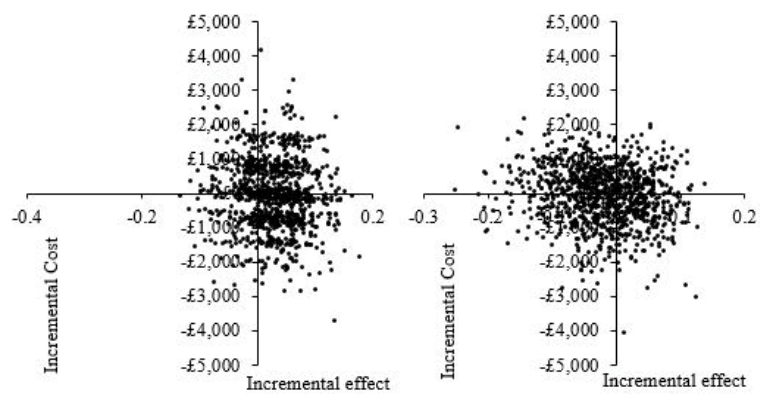

Figure 1: Cost-effectiveness plane for the QALY with 1,000 bootstrapped ICER estimates; GS v IC on the left and GM $\vee$ IC on the right.

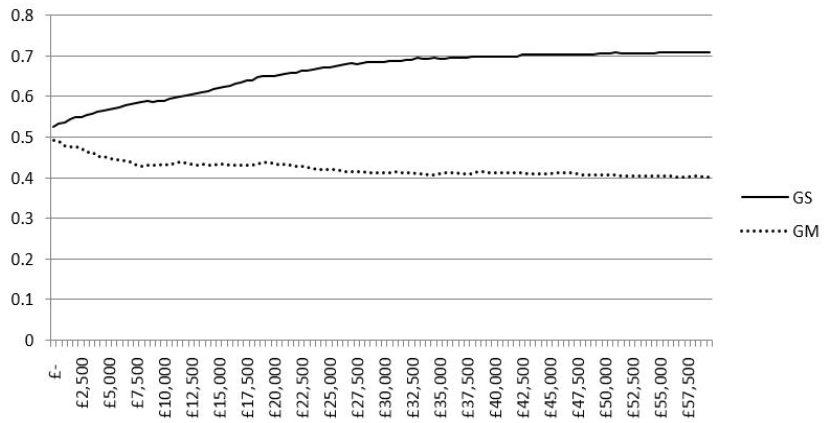

Figure 2: Cost-effectiveness acceptability curves for GS vs. IC and GM vs. IC.

delivering a community based goal-setting intervention with the aim of promoting physical and cognitive activity in older people. The Agewell centre was set up and the target study size of 75 participants to be randomised was achieved, indicating that the study was feasible. Attendance at the centre was good, with participants attending a mean of 34 activity sessions over the year. Furthermore, at 12 month followup 70 participants were assessed, which is a retention rate of $93 \%$. The sample size for cost-effectiveness analysis is often smaller than the full clinical effectiveness sample due to fewer participants providing complete information on health and social care resource use. In this study, all 70 participants in the clinical effectiveness sample completed the resource use questionnaire and were used in the cost-effectiveness analysis. Health and social care costs did not differ significantly between the control and goal-setting conditions over 12 months, and the incremental cost between conditions was low meaning that the intervention is unlikely to have affected participants' patterns of health and social care use in the short-term.

The partnership with Age Cymru Gwynedd a Môn (ACGM) and the involvement of the volunteer management group of centre attendees was key to the success of the study. The program of activities offered at the Agewell Centre was developed following consultation with centre attendees, encouraging engagement between participants and the centre. A co-ordinator was employed by the centre, and trained volunteers provided additional support. Attendance records for the centre indicate that up to 200 people could attend the centre annually. The annual cost of running the centre was estimated to be $£ 38,122$ in total or $£ 191$ per attendee (including non-trial attendees). In comparison to centre set-up and running costs, the delivery of the intervention was low cost as it only required the additional staff time involved in conducting the initial goal-setting interviews and subsequent mentoring calls.

The findings from the clinical effectiveness analysis in Clare et al. [9] showed that participants in all three arms of the pilot study benefitted across a range of outcome measures; however a specific focus on identifying individual behaviour change goals in a goal-setting interview was required to achieve increased activity engagement and greater benefits in cognition, health, diet and fitness. This paper explored the cost-effectiveness of the intervention and found that goalsetting is likely to be cost-effective compared to the control condition; however, while the addition of mentoring is likely to be effective, it is unlikely to be cost-effective. The goal-setting interviews were carried out by a researcher; however a sensitivity analysis was undertaken exploring the effect of the interviews being carried out by an ACGM staff member on a lower salary. The sensitivity analysis supported the base case analysis, in that goal-setting was likely to be cost-effective compared to the control condition.

\section{Conclusion}

The preliminary cost-effectiveness results indicate a high probability that the goal-setting intervention is cost-effective compared to the control condition at a threshold of $£ 20,000-£ 30,000$ per QALY gained. However, due to the small sample size and the QALY differences between groups not being statistically significant, it is not possible to conclusively state that the intervention was cost-effective compared to control, and a larger scale study is required. Communitybased interventions aimed at increasing physical, cognitive and social activity in older people may have a beneficial effect on health, so lowcost interventions such as the Agewell pilot study have an important role to play in keeping people active in the community.

\section{Acknowledgement}

This study was funded by the Lifelong Health and Well-being Programme through the Medical Research Council (G1001888/1). We acknowledge the support of Age Cymru Gwynedd a Môn and would like to thank the volunteers at the Nefyn Agewell Centre, and all the members of the Nefyn Agewell Centre especially those who took part in the research project.

\section{References}

1. Office for National Statistics (2013)

2. McCrae N, Murray J, Banerjee S, Huxley P, Bhugra D, et al. (2005) 'They're all depressed, aren't they?' A qualitative study of social care workers and depression in older adults. Aging and Mental Health 9: 508-516.

3. Victor CR, Scrambler SJ, Bowling A, Bond J (2005) The prevalence of, and risk factors for, loneliness in later life: A survey of older people in Great Britain. Ageing and Society 25: 357-375.

4. Zaninotto $P$, Faalaschetti E, Sacker E (2009) Age trajectories of quality of life among older adults: results from the English Longitudinal Study of Ageing. Quality of Life Research 18: 1301-1309.

5. Wilson DM, Harris A, Hollis V, Mohankumar D (2010) Upstream thinking and health promotion planning for older adults at risk of social isolation. International Journal of Older People Nursing 6: 282-288.

6. Berkman LF, Glass TA, Brisette I, Seeman TE (2000) From social integration to health: Durkheim in the new millennium. Social Science and Medicine 51 843-57.

7. Fratiglioni L, Paillard-Borg S, Winblad B (2004) An active and socially integrated lifestyle in late life might protect against dementia. The Lancet Neurology 3 : 343-353.

8. Hobbs N, Godfrey A, Lara J, Errington L, Meyer TD, et al. (2013) Are behaviora interventions effective in increasing physical activity at 12 to 36 months in adults aged 55 to 70 years? A systematic review and meta-analysis. BMC medicine 11: 75 
Citation: Jones CL, Edwards RT, Nelis SM, Jones IR, Hindle JV, et al (2015) Cost-Effectiveness Findings from the Agewell Pilot Study of Behaviour Change to Promote Health and Wellbeing in Later Life. Health Econ Outcome Res Open Access 1: 105. doi: 10.4172/2471-268x/1000105

Page 6 of 6

9. Clare L, Nelis SM, Jones IR, Hindle JV, Thom JM, et al. (2015) The Agewell trial: a pilot randomised controlled trial of a behaviour change intervention to promote healthy ageing and reduce risk of dementia in later life. BMC Psychiatry 15: 25

10. Clare L, Hindle JV, Jones IR, Thom JM, Nelis SM, et al. (2012) The AgeWell study of behavior change to promote health and wellbeing in later life: study protocol for a randomized controlled trial. Trials 13:115

11. EuroQoL Group (1990) EuroQoL- a new facility for the measurement of healthrelated quality of life. Health Policy 16: 199-208.

12. Dolan P (1997) Modeling valuations for EuroQol health states. Med Care 35:1095-1108

13. Ridyard CH, Hughes DA (2012) Development of a Database of Instruments for Resource-Use Measurement: Purpose, Feasibility, and Design. Value in Health 15: 650-655.
14. Curtis L (2012) Unit Costs of Health and Social Care. UK: Personal Social Services Research Unit, University of Kent, Canterbury.

15. Department of Health (2012) NHS Reference Costs 2011-12.

16. Medical Research Council (2008) Complex interventions guidance.

17. Edwards RT, Hounsome B, Linck P, Russell IT (2008) Economic evaluation alongside pragmatic randomised trials: developing a Standard Operating Procedure for clinical trials units. Trials 14: $64-65$.

18. Briggs A, Wonderling D, Mooney C (1997) Pulling cost-effectiveness analysis up by its bootstraps: A non-parametric approach to confidence interval estimation. Health Economics. 6: 327-340.

19. Kind P, Hardman G, Macran S (1999) UK Population Norms for EQ-5D. York: Centre for Health Economics, University of York.

20. National Institute for Health and Clinical Excellence (2013) Guide to the methods of technology appraisal. London: National Institute for Health and Clinical Excellence. 